\title{
Remarks on the Quantum Field Theory in Lattice Space. II
}

\author{
Hiroshi Ezawa \\ Department of Physics, Gakushuin University, Mejiro, Toshima-ku, Tokyo
}

Received July 24, 1967

\begin{abstract}
We consider a strong-coupling approach to $\lambda \phi^{4}$-meson theory as formulated in a lattice space which is of simple cubic type having lattice constant $a$ and total volume $V$. Self-adjointness and regularity of the Hamiltonian are established. The strong-coupling perturbation series are examined for the cases with and without mass renormalization. The series for ground state and oneparticle state as well as for their energies are shown to converge when the coupling constant is sufficiently large, say $\lambda>\lambda_{c}$ (sufficient condition). The bounds $\lambda_{c}$ we have found increase with the total volume $V$ and/or the cut-off momentum $a^{-1}$. Some other features of the strong-coupling perturbation theory are also discussed.
\end{abstract}

\section{Introduction}

This paper is to give an account of our attempt to study the perturbation theory as applied to a quantum field theory in lattice space.

The field is defined at each site of a simple cubic lattice, and it may be regarded as an average of local field over the corresponding unit cell. ${ }^{1}$ While in the previous paper [2] we studied cases of uncoupled lattice sites, we now wish to try the strong-coupling perturbation theory $[3,4]$ to take care of the coupling which in fact is caused by gradient terms necessarily present in relativistic Hamiltonians. Taking up the (lattice space version of) neutral scalar field $\phi$ with $\lambda \phi^{4}(\lambda \gg 1)$ interaction [4], we shall use Kato's theorems (1) to establish self-adjointness of the perturbed Hamiltonian and (2) to examine convergence of the perturbation series $[5,6]$.

It is indeed remarkable that, if one agrees to keep the cut-off momentum as well as the volume of the "world" finite, then in the strongcoupling scheme KaTO's theory is applicable to the $\lambda \phi^{4}$ theory, for in the weak-coupling scheme magnitudes of unperturbed and perturbation Hamiltonians cannot be compared in Kato's sense.

As for the latter scheme one might recall that A. M. JAFFE studied the model in two-dimensional space-time to prove that the weak-coupling perturbation series for Green functions are all divergent [7]; each term in the series being finite, though, in the world with such a low dimen-

${ }^{1}$ It may be recalled that YUKAwA proposed a hypothesis that the space consists of "elementary domains" which are not divisible any further [1]. 
sionality. We note in parentheses that his expansion was in powers of the bare coupling constant $\lambda$ and no renormalizations were made as to the mass and wave functions also.

The following argument will be enough to let one expect divergence of perturbation series also in four-dimensional space-time; here of course momentum cut-off has to be introduced to keep each term in the series finite: Suppose the series were convergent for a coupling constant $\lambda>0$, then as power series they should converge for $\lambda^{\prime}=-\lambda<0$ also. But, this cannot be the case because the theory with the negative coupling constant should have no ground state. In fact, C. S. LAM found a singularity of $\log \lambda$ type when calculated Green functions by applying the method of steepest descent to Feynman's path integral [8]. The failure of the weak-coupling perturbation theory provides another motivation for attempting at the strong-coupling approach.

In Sec. II Hamiltonian for the $\lambda \phi^{4}$ model in lattice space (simple cubic, lattice constant $a$, total volume $V$ ) will be set up and perturbation Hamiltonian will be separated from the gradient term, i.e. an lattice space analog of $2^{-1} \int(\nabla \phi)^{2} d^{3} x$. Then in Sec. III we establish Kato's fundamental inequality for the present model thereby proving the selfadjointness and regularity of the perturbed Hamiltonian. These properties will be used in Sec. IV to find out a bound $\lambda_{c}$ such that the perturbation series for ground state and one-particle states be convergent for $\lambda>\lambda_{c}$. The result depends on whether or not the mass is renormalized. If in the case of the renormalized mass one wishes to let $a \rightarrow 0$, then the bound $\lambda_{c}$ increases towards infinity. The same thing happens if one let $V \rightarrow \infty$. It will be shown in Sec. $V$ that such difficulties cannot be avoided in the present perturbation scheme no matter how we improve the estimates concerning KaTo's inequality. It has to be remarked, however, that $\lambda>\lambda_{c}$ is a sufficient condition but may not be necessary.

\section{Hamiltonian}

We wish to set up a lattice space analog of the Hamiltonian,

$$
\mathscr{H}_{\text {cont }}=\int d^{3} x \frac{1}{2}\left[\pi^{2}+(\nabla \phi)^{2}+\mu_{0}^{2} \phi^{2}+\lambda \phi^{4}\right], \quad(\lambda \gg 1) .
$$

We take a simple cubic lattice (lattice constant $a$, total volume $V$ ) to each site $\boldsymbol{s}$ of which field variables, $\pi(\boldsymbol{s})$ and $\phi(s)$, are assigned. They obey

$$
[\pi(\boldsymbol{r}), \phi(\boldsymbol{s})]=-i a^{-3} \delta_{\boldsymbol{r}, \boldsymbol{s}}, \quad \text { etc. }
$$

As in the previous paper in which the gradient term $\frac{1}{2}(\nabla \phi)^{2}$ was completely ignored, we use new variables, $p_{\boldsymbol{s}}$ and $q_{\boldsymbol{s}}$, defined by

$$
\left.\begin{array}{l}
\pi(s)=\eta^{1 / 2} a^{-3 / 2} p_{s} \\
\phi(s)=\eta^{-1 / 2} a^{-3 / 2} q_{s}
\end{array}\right\}
$$


with a $c$-number $\eta$ fixed shortly. They obey canonical commutation relations

$$
\left[p_{\boldsymbol{r}}, q_{\boldsymbol{s}}\right]=-i \delta_{\boldsymbol{r}, \boldsymbol{s}}, \text { etc. }
$$

Now, the space integral in (2.1) is replaced by a lattice sum and the gradient term by ${ }^{2}$

$$
(\nabla \phi)^{2}=\sum_{i=1}^{3}\left[\phi\left(s+\boldsymbol{a}_{i}\right)-\phi(s)\right]^{2} / a^{2},
$$

where $\boldsymbol{a}_{i}$ 's are primitive lattice vectors, $\left|\boldsymbol{a}_{i}\right|=a$. In the following, convention will be adopted to suppress the subscript $i$ of $\boldsymbol{a}_{i}$ and to write $\sum_{i}$ as $\sum_{\boldsymbol{a}}$. Then, in terms of the new variables, the lattice Hamiltonian becomes

where

$$
\mathscr{H}=\eta H_{\varkappa} ; \quad H_{\varkappa}=H_{0}+\varkappa H^{\prime},
$$

and

$$
\begin{aligned}
& H_{0}=\sum_{\boldsymbol{s}} h_{0 \mathbf{s}} ; \quad h_{0}=\frac{1}{2}\left(p^{2}-v q^{2}+q^{4}\right)-c, \\
& H^{\prime}=-\sum_{\boldsymbol{s}} \sum_{\boldsymbol{a}} q_{\boldsymbol{s}} q_{\boldsymbol{s}+\boldsymbol{a}},
\end{aligned}
$$

$$
\begin{aligned}
\varkappa & =\lambda^{-2 / 3}, \quad \eta=a^{-1} \lambda^{1 / 3}, \\
\nu(\lambda) & =-\lambda^{-2 / 3}\left[6+\mu_{0}^{2} a^{2}\right] ;
\end{aligned}
$$

the constant $c$ is determined such that the lowest eigenvalue $\varepsilon_{0}$ of $h_{0}$ vanishes. The subscripts $\boldsymbol{s}$ in $h_{0 s}$ are suppressed; this convention will be followed whenever there is no confusion.

It may be noticed in passing that only the choice $\eta \infty a^{-1}$ can give $H_{0}$ and $H^{\prime}$ finite forms, by which we mean polynomials in $p_{\boldsymbol{s}}$ and $q_{\boldsymbol{s}}$ whose coefficients remain finite when $a \rightarrow 0$; such a choice of $\eta$ is possible only for renormalizable theories. $\lambda$-dependence of $\eta$ is taken as such just for the sake of convenience.

\section{Self-Adjointness and Regularity of $\boldsymbol{H}_{\boldsymbol{x}}$}

The notion of self-adjointness is well-known. An operator $H_{x}$ is said to be a regular function of $x$ if its resolvent

$$
R_{x}(z)=\left(H_{\varkappa}-z\right)^{-1}, \quad(z: \text { complex number })
$$

is regular in a neighborhood of $x=0$ for some fixed $z=\hat{z}$. The following discussion will be based upon the

Theorem 1. (F. ReLLICH [9], T. Kato [5, 6]): Let $H_{x}$ be formally given by $H_{x}=H_{0}+x H^{\prime}$, where $H_{0}$ and $H^{\prime}$ are symmetric and have domain $\mathfrak{D}$ dense in a Hilbert space $\mathfrak{Y}$. Let $H_{0}$ be bounded below and let there be con-

${ }^{2}$ There are several different ways to set up the lattice space version of the gradient term. For long-wave excitations they do lead to essentially the same results. See Ref. [3] and [4]. 
stants, $A$ and $B$, such that

$$
\left|\left\langle\Psi, H^{\prime} \Psi\right\rangle\right| \leqq A+B\left\langle\Psi, H_{0} \Psi\right\rangle
$$

for every normalized $\Psi$ in $\mathfrak{P}$, where $B \geqq 0$ but $A$ may be negative. Then for $|x|<B^{-1}, H_{\varkappa}$ has a self-adjoint extension (FRIEDRICH's extension [10]), which is a regular function of $\varkappa$.

In order to apply this theorem to our Hamiltonian $H_{x}$, we have to remove its $\varkappa$-dependence through $\lambda$ of $\nu(\lambda)$ thereby making the $x$ dependence of $H_{x}$ come solely from that $x$ in front of $H^{\prime}$ in accordance with KaTo's scheme. Supposing that we are interested in the selfadjointness and the convergence problem at a particular value $\hat{\lambda}$ of the coupling constant, let us fix that $\lambda$ in $v(\lambda)$. Namely we set $\hat{v}=v(\hat{\lambda})$.

Let the Hilbert space $\mathfrak{F}$ be a $N$-fold direct product the space of $L_{2}$ functions of $q_{i}\left(i=1,2, \ldots, N=V / a^{3}\right)$ and the common domain $\mathfrak{S}$ of $H_{0}$ and $H^{\prime}$ be constituted of all the finite linear combinations of $N$-dimensional harmonic oscillator wave functions. It is obvious that both $H_{0}$ and $H^{\prime}$ are symmetric, and that $H_{0}$ is bounded below.

We now wish to establish the inequality (3.2). Let $\alpha, \beta$ and $\gamma$ be some real constants to be determined later and such that

Consider

$$
0<\alpha<1, \quad \beta>0, \quad \gamma \geqq 1 .
$$

where

$$
H_{0}-\beta H^{\prime}=\sum_{\boldsymbol{s}} h_{1 \boldsymbol{s}}+\sum_{\boldsymbol{s}} h_{2 \boldsymbol{s}}^{(+)},
$$

$$
\begin{aligned}
& h_{1 \mathbf{s}}=\frac{1}{2}\left[\alpha p_{\boldsymbol{s}}^{2}-(\nu+6 \beta \gamma) q_{\boldsymbol{s}}^{2}+q_{\boldsymbol{s}}^{4}\right]-c \\
& h_{2 \boldsymbol{s}}^{( \pm)}=\frac{1-\alpha}{2} p_{\boldsymbol{s}}^{2}+\sum_{\boldsymbol{a}} \frac{\beta}{2}\left(\gamma q_{\boldsymbol{s}}^{2}+\gamma q_{\boldsymbol{s}}^{2}+\boldsymbol{a} \pm 2 q_{\boldsymbol{s}} q_{\boldsymbol{s}+\boldsymbol{a}}\right) .
\end{aligned}
$$

Let us prove that the operator (3.4) is bounded below. First, the second half of the r.h.s. of (3.4) is the Hamiltonian of coupled harmonic oscillators. It can be uncoupled by Fourier transformation:

then,

$$
p_{\boldsymbol{s}}=N^{-1 / 2} \sum_{\boldsymbol{k}} \pi_{\boldsymbol{k}} e^{i \boldsymbol{k} \boldsymbol{s}}, \quad q_{\boldsymbol{s}}=N^{-1 / 2} \sum_{\boldsymbol{k}} \xi_{\boldsymbol{k}} e^{i \boldsymbol{k} \boldsymbol{s}},
$$

$$
\sum_{\boldsymbol{s}}^{\prime} h_{2 \boldsymbol{s}}^{(+)}=\sum_{\boldsymbol{k}}^{\prime}\left[\frac{1-\alpha}{2} \pi_{\boldsymbol{k}}^{*} \pi_{\boldsymbol{k}}+\beta \sum_{\boldsymbol{a}}^{\prime}(\gamma+\cos \boldsymbol{k} \boldsymbol{a}) \xi_{\boldsymbol{k}}^{*} \xi_{\boldsymbol{k}}\right],
$$

so that this operator is bounded below by its zero-point energy,

where

$$
\left\langle\Psi, \sum_{\mathbf{s}} h_{2 \mathbf{s}}^{(+)} \Psi\right\rangle \geqq \sqrt{(1-\alpha) \beta} N G_{+}(\gamma),
$$

$$
G_{ \pm}(\gamma)=N^{-1} \sum_{\boldsymbol{k}} \sqrt{\frac{1}{2} \sum_{\boldsymbol{a}}(\gamma \pm \cos \boldsymbol{k} \boldsymbol{a})} .
$$


We now turn to the first half of (3.4). Recall that the lowest eigenvalue of $h_{0}$ is zero. If we take the normalized ground state $\Phi_{0}(\mathbf{s})$ of $h_{1 \mathrm{~s}}$ and set $\Phi_{0}=\prod \Phi_{0}(s)$, then variation principle tells us that

$$
\left\langle\Phi_{0},\left(\sum h_{\mathbf{s}} s\right) \Phi_{0}\right\rangle \geqq N \varepsilon_{0}=0 .
$$

Hence, for any normalized vector $\Psi \in \mathfrak{P}$,

$$
\begin{gathered}
\left\langle\Psi,\left(\frac{\Sigma}{s} h_{1 s}\right) \Psi\right\rangle \geqq\left\langle\Phi_{0},\left(\sum_{\boldsymbol{s}} h_{1 \boldsymbol{s}}\right) \Phi_{0}\right\rangle \\
\geq\left\langle\Phi_{0}, \sum_{\mathbf{s}}\left(h_{1 \boldsymbol{s}}-h_{0 \mathbf{s}}\right) \Phi_{0}\right\rangle=-\left[\frac{1-\alpha}{2}\left\langle p^{2}\right\rangle_{1}+3 \beta \gamma\left\langle q^{2}\right\rangle_{1}\right] N,
\end{gathered}
$$

where

$$
\left\langle p^{2}\right\rangle_{1}=\left\langle\Phi_{0}(\mathbf{s}), p_{s}^{2} \Phi_{0}(\mathbf{s})\right\rangle, \quad\left\langle q^{2}\right\rangle_{1}=\left\langle\Phi_{0}(\mathbf{s}), q_{s}^{2} \Phi_{0}(\mathbf{s})\right\rangle
$$

are the expectation values with respect to the ground state of $h_{1 \mathbf{s}}$. Combining (3.7) and (3.9) we get

$$
\begin{gathered}
\left\langle\Psi, H^{\prime} \Psi\right\rangle \leqq \beta^{-1}\left\langle\Psi, H_{0} \Psi\right\rangle \\
+\left[\frac{1-\alpha}{2 \beta}\left\langle p^{2}\right\rangle_{1}+3 \gamma\left\langle q^{2}\right\rangle_{1}-\sqrt{\frac{1-\alpha}{\beta}} G_{+}(\gamma)\right] N .
\end{gathered}
$$

We can repeat the same argument for

$$
H_{0}+\beta H^{\prime}=\sum_{\boldsymbol{s}} h_{1 s}+\sum_{\boldsymbol{s}} h_{2 \boldsymbol{s}}^{(-)},
$$

where $h_{2 \mathrm{~s}}^{(-)}$is given by (3.6). Then, by proving that the operator is bounded below, we can get an upper bound for $-\left\langle\Psi, H^{\prime} \Psi\right\rangle$, which is the same as the right-hand side of (3.11) except for a single difference that $G_{+}(\gamma)$ is replaced by $G-(\gamma)$. However, this is only a matter of appearance because the sum over $\boldsymbol{k}$ in (3.8) can be so rearranged as to show $G_{+}=G_{-}$, which we shall write as $G$.

Thus we can conclude that the coefficents in Kato's inequality (3.2) are given by

where

$$
\begin{aligned}
A & =\left[\frac{1}{2}\left\langle p^{2}\right\rangle_{1} \varrho^{2}-G(\gamma) \varrho+3 \gamma\left\langle q^{2}\right\rangle_{1}\right] N, \\
B & =\beta^{-1},
\end{aligned}
$$

$$
\varrho=V(1-\alpha) / \beta \text {. }
$$

Thus we have shown that, in the strong-coupling scheme, the perturbation can be bounded by the unperturbed Hamiltonian in the sense of KaTo. This cannot be the case in the weak-coupling scheme.

If we now note that $\beta$ is an arbitrary positive number, the Theorem 1 tells us that for any finite $\varkappa$, our $H_{\varkappa}$ has a self-adjoint extension. This conclusion is in conformity with A. M. JAFFE's, who, by the help of the theory of partial differential equations, proved that the Hamiltonian 
(2.1) with momentum cut-off and in a finite volume is essentially selfadjoint [11]. His method of proof can readily be so modified as to apply to our lattice space Hamiltonian (2.6). We may note that, since selfadjoint extension of an essentially self-adjoint operator is unique, it should coincide with the Friedrichs extension. There will be no confusion if we denote the extension by the same notation $H_{z}$.

The Theorem 1 tells us also that the self-adjoint extension $H_{\varkappa}$ is a regular function of $\varkappa$. This property is basic to the following discussions.

\section{Convergence of Perturbation Series}

Having found that the perturbation $H^{\prime}$ can be bounded by the unperturbed Hamiltonian $H_{0}$ in the sense of (3.2), we now wish to see how much can be said about the convergence of perturbation series. We use Kato's theorems which deal with power series expansion in $\varkappa$ of projection operator $E_{*}(n)$ on the perturbed eigenspace and the associated eigenvalue that should correspond to an unperturbed eigenspace $E_{0}(n)$ having isolated eigenvalue $w_{n}^{(0)}$ (multiplicity $m<\infty$ ).

We shall consider only the ground state and the one-particle states. The former is nondegenerate. If we take advantage of the fact that the perturbation conserves momentum, we can concentrate our attention to a sector of given momentum, in which the one-particle state is nondegenerate. Since we have to fix the parameter $v$ in $H_{0}$ by $\hat{v}=v(\hat{\lambda})$ to make the $x$-dependence of $H_{x}=H_{0}+x H^{\prime}$ come solely from that $x$ in front of $H^{\prime}$, Kato's theorems give a lower bound $r_{c}(\dot{\hat{\lambda}})$ of the radius of convergence that depends on $\hat{\lambda}$. Thus, we shall get an implicit condition $\hat{\varkappa} \equiv \hat{\lambda}^{-2 / 3}<r_{c}(\hat{\lambda})$ for $\hat{\lambda}$ for which perturbation series in question is convergent.

Now, we quote the

Theorem 2. (T. Като $[5,6])$. Let $\Gamma_{n}$ be such a closed curve on complex $z$-plane that encloses $z=w_{n}^{(0)}$ in it but contains inside or on it no other point of the spectrum $\Sigma\left(H_{0}\right)$ of $H_{0}$. Then, under the assumption of Theorem 1 and for

$$
\begin{gathered}
|x|<r_{c} ; \\
r_{c}=\left\{\operatorname{Max}_{z \in \Gamma_{n}} \operatorname{Max}_{w \in \Sigma\left(H_{0}\right)}\left|\frac{A+B w}{w-z}\right|\right\}^{-1}
\end{gathered}
$$

the projection operator $E_{\varkappa}(n)$ is given by term-by-term integration of

where

$$
E_{\varkappa}(n)=-(2 \pi i)^{-1} \underset{\Gamma_{n}}{ } R_{\varkappa}(z) d z,
$$

$$
\begin{aligned}
R_{\varkappa}(z) & =\left(H_{\varkappa}-z\right)^{-1} \\
& =\left(H_{0}-z\right)^{-1}\left\{1+\sum_{p=1}^{\infty}(-\varkappa)^{p}\left[H^{\prime}\left(H_{0}-z\right)^{-1}\right]^{p}\right\} ;
\end{aligned}
$$


the resulting series is absolutely convergent and the projection $E_{*}(n)$ has the same dimensionality $m$ as $E_{0}(n)$ has.

As for the perturbed eigenvalues, the assumptions in Theorem 2 allow us to state the

Theorem 3. (T. Като $[5,6])$. When $m=1$, the Schrödinger perturbation series for the perturbed eigenvalue belonging to the space $E_{*}(n)$ is absolutely convergent for $x$ satisfying (4.1).

Now in our case, the spectrum of $H_{0}$ consists of the points ${ }^{3}$,

$$
w_{0}^{(0)}=0, \quad w_{1}^{(0)}=\varepsilon_{1}, \quad w_{2}^{(0)}=2 \varepsilon_{1}, \ldots
$$

where $\varepsilon_{n}(\hat{v})$ is the eigenvalue of the lattice Hamiltonian $h_{0 s}$ :

$$
h_{0 s}|n\rangle_{s}=\varepsilon_{n}(\hat{v})|n\rangle_{s}, \quad \varepsilon_{0}=0<\varepsilon_{1}<\cdots .
$$

The ground state of $H_{0}$ is nondegenerate, but all the other states are highly degenerate. We note, however, that the perturbation $H^{\prime}$ conserves momentum. Since the one-particle state (energy $w_{1}^{(0)}$ ) is nondegenerate in the sector $\mathfrak{H}_{\boldsymbol{k}}$ with given momentum $\boldsymbol{k}$, we can apply the Theorem 3 to this state, too.

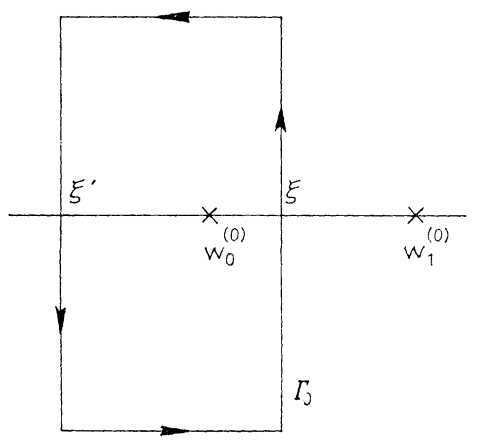

Fig. 1. Contour of integration for (4.3), $n=0$

Let us begin with the ground state. Then, the contour $\Gamma_{0}$ must enclose $w_{0}^{(0)}=0$ and no other points of $\Sigma\left(H_{0}\right)$. Choosing a rectangular $\Gamma_{0}$ as shown in Fig. 1, we wish to find its optimum position and size that yield the largest possible $r_{c}$ from (4.2); the only conditions we have for $\Gamma_{0}$ is on the positions, $\xi^{\prime}$ and $\xi$, of its intersection with the real axis,

$$
\xi^{\prime}<0 \text { and } 0<\xi<\varepsilon_{1} \text {. }
$$

If we take a sufficiently large $\Gamma_{0}$, then, for each point $w \in \Sigma\left(H_{0}\right)$, the $\operatorname{Max}_{z \in \Gamma_{0}}$ of (4.2) occurs when $z=$ real $=\xi$. Of these maximum values the $\operatorname{Max}$ is given by either one of the two points $w$ of $\Sigma\left(H_{0}\right)$ nearest to $\xi$; $\operatorname{Max}_{w \in \Sigma\left(H_{0}\right)}$

${ }^{3}$ Numerical solution of the Schrödinger equation for $h_{0}$ shows that $\varepsilon_{2}>2 \varepsilon_{1}$ irrespective of the value of $v$. 
they are $w=0$ and $w=\varepsilon_{1}$. Thus,

$$
r_{c}=\left\{\operatorname{Max}\left(\frac{A}{\xi}, \frac{A+B \varepsilon_{1}}{\varepsilon-\xi}\right)\right\}^{-1},
$$

where Max means the larger of the two quantities in the bracket; they are positive since $A, B>0$ [see (4.12) and (4.23)]. Now, it is clear that the $\xi$ is optimum when chosen in such a way that these two quantities become equal to each other. We arrive at

$$
r_{c}=\left(\tau B+\frac{2 A}{\varepsilon_{1}}\right)^{-1},
$$

where $\tau=1$ is inserted for the convenience at the next stage.

Similar considerations as applied to the one-particle state gives the same expression for $r_{c}$ with $\tau=2$. In the following we take this larger value for $\tau$ so that the resulting $r_{c}$ is good for both ground state and oneparticle state.

According to (3.12) and (3.13) the coefficients $A$ and $B$ are functions of $\alpha, \beta$ and $\gamma$ which we may vary. Our task is now to find out the optimum set of values for these parameters that yields the largest possible $r_{c}$. It is more convenient to use the set $\varrho, \beta$ and $\gamma$; then $B$ depends on $\beta$ only and $A$ depends on $\varrho$ explicitly, on $\gamma$ through $G(\gamma)$ and finally on $\hat{v}_{1}=\hat{v}+6 \beta \gamma$ through the matrix elements involved in (3.12).

First, we vary $\varrho$, which can be done without affecting $B$. From (3.12) we know that $A$ takes on the minimum value,

$$
A=\left(M_{1}+M_{2}\right) N /\left\langle p^{2}\right\rangle_{1}
$$

when $\varrho=2 G(\gamma) /\left\langle p^{2}\right\rangle_{1}$, where

$$
\begin{aligned}
& M_{1}=3 \gamma \Delta\left(\hat{v}_{1}\right), \quad \Delta \equiv\left\langle p^{2}\right\rangle_{1}\left\langle q^{2}\right\rangle_{1}-\frac{1}{4} ; \\
& M_{2}=\frac{3}{4} \gamma-G(\gamma)^{2}\left[\sim(16 \gamma)^{-1} \text { for } \gamma \gg 1\right] .
\end{aligned}
$$

Numerical evaluation of $G(\gamma)$ shows that its asymptotic form given in (4.11) is fairly good already for $\gamma=2$ (see Table 1 ).

Table 1

\begin{tabular}{l|ll|l}
\hline \multirow{3}{*}{$3 \gamma$} & \multicolumn{2}{|c|}{$G(\gamma)$} & $\frac{3}{2} \gamma-G(\gamma)^{2}$ \\
\cline { 2 - 4 } & $N=6^{3}$ & $N=10^{3}$ & $N=6^{3}$ \\
\hline 3.0 & 1.1931 & 1.1937 & 0.0765 \\
3.5 & 1.3000 & 1.3000 & 0.0600 \\
6.0 & 1.7227 & 1.7227 & 0.0322
\end{tabular}

The optimum choices of $\beta$ and $\gamma$ depend on whether or not we make mass renormalization. 
(i) Case of No Mass-Renormalization. Because in (4.8) $A$ is proportional to the big number $N=V / a^{3}$ and $B$ is inversely proportional to $\beta$, we can expect that the optimum $\beta$ will turn out to be very small. Then, we may assume $6 \beta \gamma \ll 1$ so that we can vary $\gamma$ keeping $\hat{v}_{1} \sim \hat{v}$ and consequently $\Delta\left(\hat{v}_{1}\right)$ almost unaffected; the subscript 1 of $\left\langle p^{2}\right\rangle_{1}$ can be dropped and the angular bracket shall mean the expectation value with respect to the ground state of $h_{0}$. In such a circumstance, $A$ in (4.9) will take on the minimum value,

when

$$
A=\frac{1}{2} \sqrt{3} \Delta N /\left\langle p^{2}\right\rangle
$$

$$
\gamma=(4 \sqrt{3} \Delta)^{-1}
$$

With (4.12), the lower bound for the radius of convergence becomes

$$
r_{c}=\left(\frac{2}{\beta}+\frac{\sqrt{3 \Delta}}{\varepsilon_{1}\left\langle p^{2}\right\rangle} N\right)^{-1}
$$

Of the two terms in the bracket here, the second one is almost independent of $\beta$. If we take such a $\beta$ that makes the first term equal the second, then the underlying assumption of small $\beta$ is in fact satisfied:

$$
6 \beta \gamma=N_{0} / N \ll 1, \quad\left(N_{0} \equiv \varepsilon_{1}\left\langle p^{2}\right\rangle / \Delta\right),
$$

for sufficiently large $N$. For such a choice of $\beta$, our condition for the convergence, $\hat{\varkappa}<r_{c}(\hat{\lambda})$, becomes

$$
\lambda>\lambda_{c} \quad \text { with } \quad \lambda_{c}^{2 / 3}=\frac{2 \sqrt{3} \Delta}{\varepsilon_{1}\left\langle p^{2}\right\rangle} N,
$$

where the carrets over $\lambda$ etc. have been omitted.

From this result we observe first of all that limit $a \rightarrow 0$ and/or $V \rightarrow \infty$ cannot be taken without the catastrophe $\lambda_{c} \rightarrow \infty$. This is because our $A$ is proportional to $N=V / a^{3}$. That this is unavoidable in the present scheme will be explained in the last section.

In Table 2, there are given two sets of numerical data pertinent to our discussion which are obtained by solving the Schrödinger equation for $h_{0}$ numerically.

Table 2

\begin{tabular}{r|rlllrl}
\hline$v$ & $\varepsilon_{1}$ & $\left\langle p^{2}\right\rangle$ & $\left\langle q^{2}\right\rangle$ & $\Delta$ & $N_{0}$ & $\lambda^{2 / 3} / N$ \\
\hline & & & & & & \\
0 & 1.36967 & 0.706900 & 0.362019 & 0.0059 & 160 & 0.26 \\
-3 & 2.08583 & 1.047614 & 0.239390 & 0.00079 & 2800 & 0.044
\end{tabular}

Now, if our lattice constant should be chosen to be smaller than the Compton wave length corresponding to the bare mass, i.e.,

$$
\mu_{0} a \lesssim 1
$$

then the strong coupling would imply

$$
-\hat{v}=\hat{\lambda}^{-2 / 3}\left(6+\mu_{0}^{2} a^{2}\right) \ll 1,
$$


in which case the pertinent numerical data are given by the first row of Table 2. If $N=10^{3}$ for instance, then the $\lambda_{c}$ turns out to be 2600 , such a big number!

The second row of Table 2 suggests that the situation could be improved slightly if $\hat{v}$ were large negative; this is due to the small $\Delta$, which, being the difference between the uncertainty product squared and its harmonic oscillator limit $1 / 4$, decreases with increasing strength of the harmonic term $-(1 / 2) \hat{v} q^{2}$ in $h_{0}$. But, (4.18) tells us that $-\hat{v} \gtrsim 1$ implies a very coarse cell division, $\mu_{0} a \gg 1$.

(ii) Case of Renormalized Mass. Unfortunately, mass renormalization can be discussed only by taking a recourse to lower order perturbation calculations. If $\hat{\lambda}$ is so large that $\hat{x} \ll r_{c}$, then we may content ourselves with the first order approximation, in which we get

$$
\omega(\boldsymbol{k})^{2}=\mu^{2}+\sigma \boldsymbol{k}^{2}, \quad(k a \ll 1),
$$

for the energy of one-particle state with momentum $\boldsymbol{k}$. For $\lambda=\hat{\lambda}$,

$$
\begin{aligned}
\mu^{2} & =a^{-2}\left[\lambda^{2 / 3} \varepsilon_{1}^{2}-12 \varepsilon_{1}|\langle 1|q| 0\rangle|^{2}\right], \\
\sigma & =2 \varepsilon_{1}|\langle 1|q| 0\rangle|^{2} .
\end{aligned}
$$

The mass renormalization means to determine the counterterm $\Delta \mu^{2}$ in $\mu_{0}^{2}=\mu^{2}-\Delta \mu^{2}$ such that the physical mass $\mu$, and not the bare mass $\mu_{0}$, is kept fixed when $a$ approaches zero. We recall that $\varepsilon_{1}$ and $\langle 1|q| 0\rangle$ depend on the parameter $\hat{v}$ which involves $\Delta \mu^{2}$ now. The mass renormalization $\Delta \mu^{2}$ makes $\hat{v}$ positive, so that the potential function of $h_{0}$ is now $W$-shaped with the depth of the valleys being proportional to $\hat{v}^{2}$. Therefore, $\varepsilon_{1}$ decreases exponentially fast with increasing $\hat{v}$ and $\langle 1|q| 0\rangle$ grows up very slowly. The renormalization condition then amounts to requiring that $\varepsilon_{1}$ be a small quantity of order $\hat{\lambda}^{-2 / 3}$ for large $\hat{\lambda}$ (see (4.20)) and therefore that

$$
\hat{v} \gg 1 \text {. }
$$

The valleys of the potential function are so deep that the lowest two levels become almost degenerate (Fig. 2). Such a case can be handled by using the resonance approximation [12]. See Appendix.

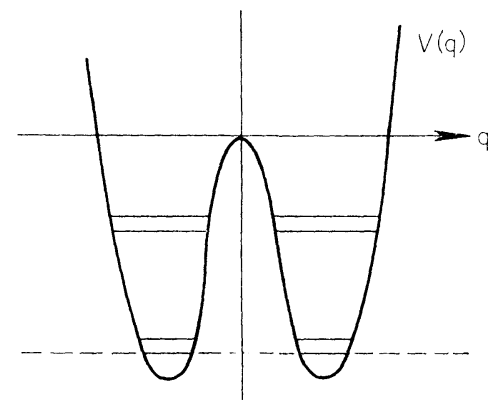

Fig. 2. For the potential $V(q)$ having deep valleys symmetric with respect to the origin, the energy spectrum has a doublet structure 
Now, we come back to (4.9). Taking $\left\langle p^{2}\right\rangle_{1}$ and $\left\langle q^{2}\right\rangle_{1}$ from Appendix, we find that $A$ takes on its minimum value,

$$
A=\frac{3}{2}(\hat{v}+6 \beta) N,
$$

when $\gamma$ is the smallest, $\gamma=1$. Then, choosing $\beta$ appropriately we make (4.8) maximum, obtaining

$$
r_{c}=\left[\frac{3 \hat{v}}{\varepsilon_{1}} N+12 \sqrt{\frac{N}{2 \varepsilon_{1}}}\right]^{-1} .
$$

Thus for large $N$, the sufficient condition for convergence becomes:

$$
\lambda^{2 / 3}=\frac{(\mu a)^{2}+12 \varepsilon_{1}|\langle 1|q| 0\rangle|^{2}}{\varepsilon_{1}^{2}}>\frac{3 v}{\varepsilon_{1}(v)} N
$$

the carrets are again removed and substitution is made for $\lambda^{2 / 3}$ from (4.20).

Now, we have to distinguish two cases. First, if $a$ was chosen so small that $(\mu a)^{2}$ be neglected in (4.25), then substitution of (A.9) would lead to an absurdity, $N<2$, for any value of $\lambda$. Unfortunately therefore, taking the continuum limit $a \rightarrow 0$ should be out of our scope again.

If on the contrary, the lattice constant $a$ is fixed and if $v$ is taken so large that $(\mu a)^{2}$ dominates in (4.25), then the equality part gives,

$$
\varepsilon_{1}(\nu)=\mu a / \lambda^{1 / 3},
$$

which relates $v$ and $\lambda$. The inequality part becomes

$$
\lambda>[3 \nu N /(\mu a)]^{3} .
$$

The combination of these two constitutes our convergence condition.

We know from (4.26) as combined with (A.8) that $\nu$ is a slowly varying function of $\lambda^{1 / 3} /(\mu a)$ :

$$
\lambda^{1 / 3} /(\mu a)=\frac{4}{3} v^{-2} \exp \sqrt{\nu^{3} / 2}, \quad[\equiv f(\nu)] .
$$

Substitution of this $\lambda$ into (4.27) yields

$$
\frac{N}{(\mu a)^{2}}<\frac{4}{9} \nu^{-3} \exp \sqrt{\nu^{3} / 2}, \quad[\equiv g(\nu)] .
$$

In this form, the question of finding the bound $\lambda_{c}$ can be solved graphically. See Fig. 3. For a given set of $N$ and $\mu \alpha$, find $\nu$ that satisfies (4.29) with its inequality sign replaced by equality sign. Then,find the corresponding $\lambda$ by (4.28) and this is the desired $\lambda_{c}$. The perturbation series we have been considering are convergent for $\lambda>\lambda_{c}$. 


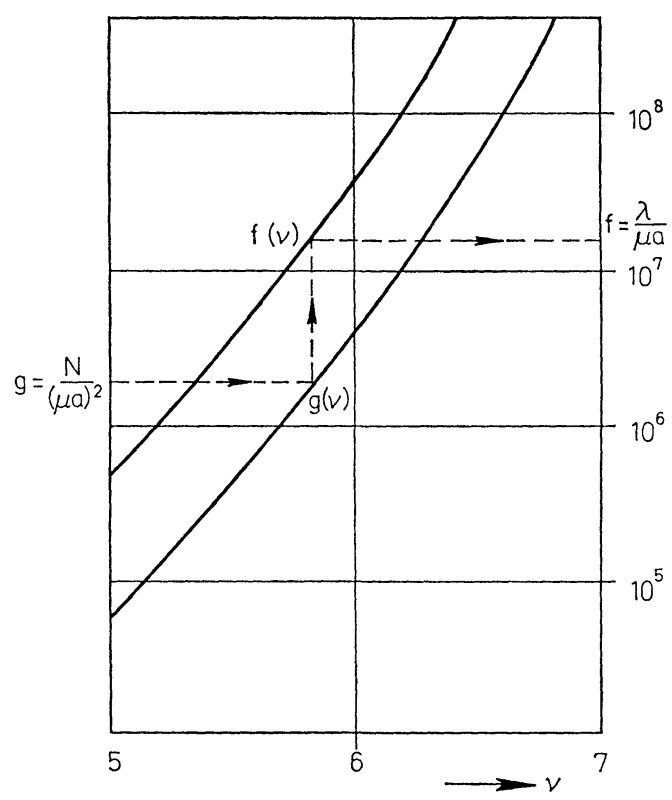

Fig. 3. Nomogram to find $\lambda_{c}$ for given values of $N$ and $\mu a$. The case of renormalized mass

\section{Discussion}

We have shown that, in the strong-coupling scheme [4], Kato's theory $[5,6]$ is applicable to the $\lambda \phi^{4}$ theory to prove the selfadjointness of the Hamiltonian. As is well-known, this cannot be the case in the weak-coupling scheme.

We have shown also that the strong-coupling perturbation series for the ground state and the one-particle states are convergent if the coupling constant is sufficiently large, $\lambda>\lambda_{c}$ with the bound $\lambda_{c}$ given by (4.16) for the case of no mass-renormalization and by Fig. 3 for the case of renormalized mass. It is annoying, however, that the bound increases towards infinity with the cut-off momentum $a^{-1}$ as well as with the volume $V$ of the "world".

As long as we use KATO's theory, which was originally designed for systems in atomic physics having finite numbers of degrees of freedom, to discuss the convergence in our field theoretical problem, the difficulty will remain no matter how we improve the estimates of $A$ and $B$ in the inequality (3.2), because $A$ is inevitably proportional to $N=V / a^{3}$. This is most easily seen by taking a vector of the form,

4 Commun. math. Phys., Vol. 9

$$
\Psi=\prod_{\mathbf{s}} \frac{|0\rangle_{s}+g|1\rangle_{s}}{\sqrt{1+g^{2}}}
$$


where $g$ is a small number. With this vector we have

$$
\left\langle\Psi, H_{0} \Psi\right\rangle=w_{0}^{(0)}+O\left(g^{2}\right),
$$

while the expectation value of the perturbation,

$$
\left\langle\Psi, H^{\prime} \Psi\right\rangle=-\sum_{\boldsymbol{s}, \boldsymbol{a}} \frac{2 g}{1+g^{2}}|\langle 1|q| 0\rangle|^{2},
$$

is $O(g)$ and is proportional to $N$. Hence $A \infty N$ if Kato's inequality (3.2) is to be valid for $\Psi$ 's dense in a Hilbert space $\mathfrak{S}$.

Our result does not imply that the perturbation series do diverge when $\lambda>\lambda_{c}$; KATO's theory provides only a sufficient condition for convergence. Yet, one may notice that variety of intermediate states in higher order perturbation will increase with $N$ so that the slower the convergence may become the larger the number $N$ is.

In the case of no mass-renormalization one has to keep the cut-off momentum $a^{-1}$ finite. One can take a finite $V$ if one is interested in local observables in a finite time interval. Yet, there arises an unsatisfactory feature when one examines the physical mass from the first order perturbation calculation. For $N$ and $\lambda$ so large as to satisfy the requirements, (4.15) and (4.16) respectively, the formula (4.20) gives $\mu a \gg 1$, that is, the Compton wave length with the physical mass turns out necessarily to be much smaller than the lattice constant. Such a coarse cell division will be meaningless when one is interested in the structure of self-field in the one-particle states. The situation will be improved if we get better estimate of $A$ so that we may deal with the smaller $\lambda$.

In the case of the renormalized mass, there is no such difficulty. However, the large value of $v$ necessitated by the condition of massrenormalization makes the $\sigma$ in (4.21) much smaller than 1 , so that the one particle energy (4.19) loses the relativistic covariance. One may recall that ScHIFF [4] argued that, for the case of no mass-renormalization and of $v=0$, the higher order corrections would solve the problem of covariance ${ }^{4}$. But, it seems doubtful if an argument like his can be given for the present case of $\nu \gg 1$. We would rather suggest the following: The convergence of the perturbation series is particularly slow in this case because the mass-renormalization requires the distance between the lowest two levels of $H_{0}$ to be so small. Thus, the situation will be improved if one can take that part $H_{1}^{\prime}$ of perturbation which connects those two levels to treat $H_{01}=H_{0}+x H_{1}^{\prime}$ as a new unperturbed Hamiltonian. From this point of view, essentially, each lattice site can take on only two states, so that the field in the lattice space is equivalent to a system of spins $(s=1 / 2)$ sitting at each lattice site and interacting with the nearest neighbors through $\varkappa H_{1}^{\prime}$. We can hope to solve the eigen-

${ }^{4}$ In this case, the lowest order value of $\sigma$ is 0.9888 . 
value problem of this Hamiltonian $H_{01}$ by invoking the highly developed theories of spin waves. If this is done, the remaining perturbation effect will be suppressed because the transitions due to $H^{\prime}-H_{1}^{\prime}$ involve large energy differences. Further discussions will be given in subsequent papers.

\section{Appendix}

We use the resonance approximation [12] to determine the lowest two levels of a particle (mass $=1$ ) moving in the potential,

$$
V(q)=\frac{1}{2}\left(-v q^{2}+q^{4}\right) \quad(v \gg 1) .
$$

This potential has two deep valleys $V_{ \pm}(q)$ that can be approximated by

$$
V_{ \pm}(q)=-\frac{1}{8} \nu^{2}+\nu(q \mp \bar{q})^{2}, \quad\left(\bar{q}=\sqrt{\frac{\nu}{2}}\right) .
$$

In the zeroth approximation, therefore, the lowest state is two-fold degenerate, with harmonic oscillator wave functions,

$$
u_{ \pm}(q)=\left(\frac{2 \bar{q}}{\pi}\right)^{1 / 4} \exp \left[-\bar{q}(q \mp \bar{q})^{2}\right],
$$

one localized in the right valley and the other in the left valley.

Once one takes their coupling through their tails, the degeneracy is lifted and the eigenstates become:

$$
\left.\begin{array}{l}
u_{0}(q) \\
u_{1}(q)
\end{array}\right\}=[2(1 \pm S)]^{-1 / 2}\left\{u_{+}(q) \pm u_{-}(q)\right\},
$$

where $S$ is the overlap integral,

$$
S=\int u_{+} u_{-} d q=\exp \left[-\sqrt{\frac{\nu^{3}}{2}}\right] .
$$

For $h_{0}=\frac{1}{2} p^{2}+V(q)$ we get

$$
\begin{aligned}
& \left\langle u_{+}, h_{0} u_{+}\right\rangle=-\frac{1}{8} \nu^{2}+\sqrt{\frac{v}{2}}+\frac{3}{16} \frac{1}{v}, \\
& \left\langle u_{+}, h_{0} u_{-}\right\rangle=\left(-\frac{1}{2} \nu^{2}+\frac{1}{4} \sqrt{\frac{\nu}{2}}+\frac{3}{16} \frac{1}{v}\right) S .
\end{aligned}
$$

Thus, the distance between the lowest two levels turns out to be

$$
\begin{aligned}
\varepsilon_{1}-\varepsilon_{0} & =\left\langle u_{1}, h_{0} u_{1}\right\rangle-\left\langle u_{0}, h_{0} u_{0}\right\rangle \\
& =\frac{3}{4}\left(v^{2}+\sqrt{2 v}\right) \exp \left[-\sqrt{\frac{v^{3}}{2}}\right],
\end{aligned}
$$

where quantities of order $S^{2}$ have been neglected. Note that this distance is called $\varepsilon_{1}$ in the text by shifting the origin of the energy scale such that $\varepsilon_{0}=0$. 
In the same approximation we have:

and

$$
\left\langle u_{1}, q u_{0}\right\rangle=\bar{q}=\sqrt{\frac{\nu}{2}},
$$

$$
\left.\begin{array}{l}
\left\langle u_{0}, q^{2} u_{0}\right\rangle=(1-S) \bar{q}^{2}+\frac{1}{4} \sqrt{\frac{2}{v}}, \\
\left\langle u_{0}, p^{2} u_{0}\right\rangle=\sqrt{\frac{v}{2}}-v^{2} S .
\end{array}\right\}
$$

Acknowledgement. Main part of this work was done while the author was visiting the II. Institut für Theoretische Physik, Universität Hamburg. He would like to thank Professor R. HaAG for the hospitality as well as for his most generous help and advices.

\section{References}

1. Yukawa, H.: Supplement to Prog. Theoret. Phys. (Kyoto) 37/38, 512 (1966).

2. Ezawa, H.: Commun. Math. Phys. (to be published).

3. Wentzel, G.: Helv. Phys. Acta 13, 269 (1940).

4. Schiff, L. I.: Phys. Rev. 92, 766 (1953).

5. Kato, T.: Journal of the Faculty of Science, Univ. of Tokyo, Sec. I, 6, (1951), Part 3.

6. - Perturbation theory for linear operators. Berlin-Heidelberg-New York: Springer 1966.

7. JAFFe, A.: Commun. Math. Phys. 1, 127 (1965).

8. LAM, C. S.: Nuovo Cimento 47, 451 (1966).

9. Rellich, F.: Math. Ann. 116, 555 (1939).

10. Friedrichs, K. O.: Math. Ann. 109, 465 (1934).

11. JAFFE, A.: Thesis. Princeton University, 1965.

12. Heisenberg, W.: Z. Phys. 40, 501 (1926).

Hiroshi Ezawa

Department of Physics

Gakushuin University

Mejiro, Toshima-ku, Tokyo/Japan 